\title{
Rhodococcus equi Pneumonia in Kidney Transplant Recipient Affected by Acute Intermittent Porphyria: A Case Report
}

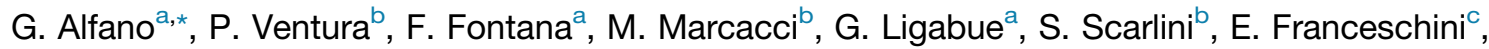 \\ M. Codeluppi ${ }^{\mathrm{c}}$, G. Guaraldi ${ }^{\mathrm{C}}$, C. Mussini ${ }^{\mathrm{C}}$, and G. Cappelli ${ }^{\mathrm{a}}$
}

${ }^{\mathrm{a}}$ Nephrology Dialysis and Transplant Unit, University of Modena and Reggio Emilia, AOU Policlinico of Modena, Modena, Italy; ${ }^{\mathrm{b}} \mathrm{Center}$ for Porphyrias, Internal Medicine 2 Unit, Department of Medical and Surgical Science for Children and Adults, University of Modena and Reggio Emilia; and 'Infectious Diseases Clinic University of Modena and Reggio Emilia School of Medicine, Department of Medicine and Medical specialities, AOU Policlinico of Modena, Modena, Italy

\begin{abstract}
Rhodococcus equi is a gram-positive coccobacillus responsible for severe infections in patients with weakened immune systems. R equi generally causes pnumonia that may evolve into fatal systemic infection if left untreated. Here, we present a case of a 67-year-old woman affected by acute intermittent porphyria (AIP) who developed $R$ equi pneumonia 7 months after kidney transplant. Although clinical features at presentation were nonspecific, lung computed tomography showed right perihilar consolidation with a mass-like appearance causing bronchial obstruction. Appropriate antibiotic including intravenous meropenem and oral azithromycin that was then switched to oral levofloxacin and oral azithromycin along with reduction of immunosuppressive therapy resolved pneumonia without provoking an acute attack of porphyria. AIP limited the choice of antibiotics for the treatment of Requi infection because some potentially porphyrinogenic antibacterial agents were avoided. Based on this experience, azithromycin and meropenem can be safely administered for the treatment of $R$ Equi infection in patients with AIP.
\end{abstract}

$\boldsymbol{R}$ HODOCOCCUS equi is a gram-positive coccobacillus responsible for zoonotic infections expressing mainly as bronchopneumonia, abscess, lymphadenitis, or ulcerative enterocolitis [1]. The bacterium has a worldwide distribution; its presence has been documented mainly in horse manure, soil, and water [2]. The first human case of $R$ equi infection presenting with cavitary pneumonia was reported in 1967 in a young man with autoimmune hepatitis on steroid therapy [3]. $R$ equi may cause potentially life-threating infections that affect predominantly immunocompromised patients affected by HIV infection and hematologic diseases or organ transplant recipients [4]. $R$ equi infection generally presents with insidious onset and typically manifests with pneumonia. To date, there is no standard treatment for this disease, and in transplant recipients treatment is based largely on the use of a long-term combination of 2 or more antibiotics.

We report a case of pneumonia due to $R$ equi infection in a kidney transplant recipient also affected by acute intermittent porphyria (AIP). We review issues regarding pathogenesis, clinical presentation, diagnosis, and treatment of
$R$ equi infection and discuss difficulties faced in choosing the best antimicrobial therapy in a patient prone to develop a potentially fatal acute attack if exposed to unsafe (porphyrinogenic) drugs.

\section{CASE DESCRIPTION}

A 67-year-old woman affected by AIP presented with nonproductive cough lasting more than 6 weeks (Fig 1). The patient had undergone kidney transplant from a deceased donor 7 months prior after having been on chronic hemodialysis treatment for 40 months. The origin of kidney failure was undetermined; the long history of hypertension associated with the likely nephrotoxic effect of porphyrin precursors was considered the main cause of her chronic kidney disease.

Disclosure: The authors declare no conflicts of interest. The authors declare that they have no competing interest.

${ }^{*}$ Address correspondence to Dr Gaetano Alfano, Specialist in Nephrology, University Hospital of Modena, Via del Pozzo, 71, 41124 Modena, Italy. Tel: +39 3665450704. E-mail: alfano. gaetano.md@gmail.com

$0041-1345 / 18$

https://doi.org/10.1016/j.transproceed.2018.02.213 


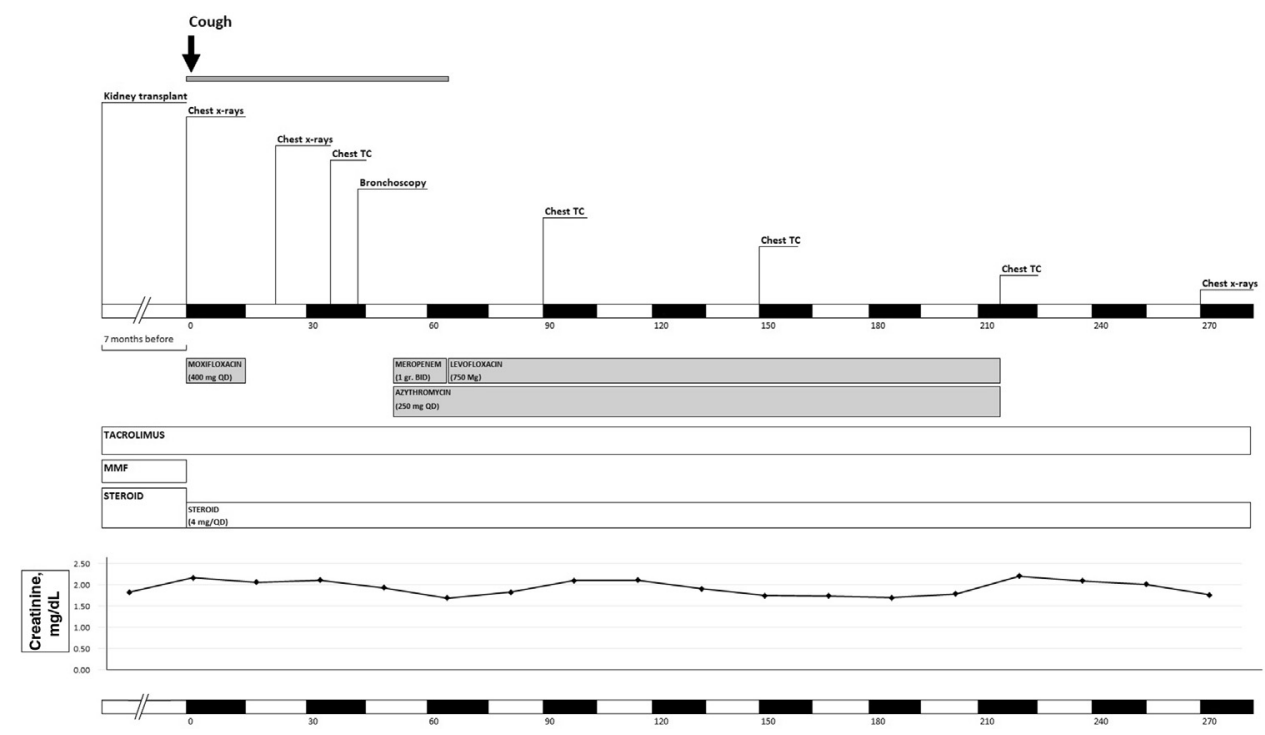

Fig 1. Timeline representing the principal investigations, antimicrobial and immunosuppressive therapy, and trend in creatinine during days 0-285 after kidney transplant. Abbreviations: AIP, acute intermittent porphyria; ALA, aminolevulinic acid dehydratase deficiency porphyria; BID, twice a day; HCP, hereditary coproporphyria; MMF, mycophenolate mofetil; QD, once a day; VP, variegate porphyria.

Her medical history was remarkable for 2 acute attacks of AIP at the age of 23 and 24 . Urinary excretion of porphobilinogen steadily elevated over time, but she did not experience other acute attacks of AIP. She was a nonsmoker with an unremarkable history of pulmonary disease. Additionally, she reported allergy to penicillin and ciprofloxacin. At the time of kidney transplant, induction with basiliximab and maintenance therapy with tacrolimus (TAC), mycophenolate mofetil (MMF), and steroid were well tolerated without triggering any acute exacerbations of AIP. Delayed graft function, paroxysmal atrial fibrillation, and development of new onset diabetes mellitus complicated the immediate postoperative period after transplant.

Given the high risk of sulfonamides to provoke an acute attack of AIP, pentamidine isethionate was used for Pneumocystis jirovecii pneumonia prophylaxis; pre-emptive therapy was used to prevent post-transplant cytomegalovirus infection.

At presentation, the patient had no signs of respiratory distress or chest pain, denied recent episodes of hemoptysis, and was apyretic. Fine crackles were clearly audible at the base of the right lung on physical examination. Laboratory data included a total leukocyte count of $8.05 \times 10^{9} / \mathrm{L}$ with a differential of $81.2 \%$ : neutrophils, $10.4 \%$; lymphocytes, $9.8 \%$; and monocytes, $7.7 \%$; hemoglobin was $11.5 \mathrm{~g} / \mathrm{dL}$. C-reactive protein level was slightly increased $(3.3 \mathrm{mg} / \mathrm{dL} ; \mathrm{N}<0.7)$. The patient showed a stable renal allograft function with an estimated glomerular filtration rate [5] of $29 \mathrm{~mL} / \mathrm{min}$. Serum transaminase, bilirubin, electrolyte, and lactate dehydrogenase values were normal. Immunosuppressive regimen included TAC (target total trough level [TTL], 7-10 ng/mL), methylprednisolone (6 mg once daily), and MMF (500 mg twice daily). Chest radiograph revealed a right lower lobe consolidation, consistent with community-acquired pneumonia. Given the stable clinical conditions with a normal hemodynamic profile, the patient was treated in the outpatient setting. A course of oral antibiotic with moxifloxacin (400 mg per day) was planned for 10 days, and MMF was stopped to favor the resolution of the presumptive bacterial pneumonia. On day 14, after the adequate course of antibiotic therapy, a second chest radiography was performed because of the persistence of a nonproductive cough. The presence of the roundish lung lesion without signs of resolution prompted the performance of high-resolution computed tomography (CT) of the lung. The CT imaging revealed a grossly right perihilar mass measuring $8.5 \times 4 \mathrm{~cm}$ that encircled and completely occluded the lumen of the right lower lobe bronchus and confirmed the smaller rounded opacity in the right lower lobe previously detected by chest radiography. Bronchoscopy revealed a broad-based, lobulated lesion arising from the right main stem bronchus and completely occluding the lumen of middle and lower lobe bronchus. Cytologic examination of cells from bronchoalveolar lavage (BAL) showed a moderate numbers of inflammatory cells, mostly macrophages; malignant cells were not found. The histologic analysis of the bronchial biopsy specimen showed a pattern of chronic granulomatous disease, accompanied by inflammatory cells of acute phase (ie, neutrophils). The histiocytes in granuloma showed a granular eosinophilic cytoplasm (von Hansemann cells) containing several bacteria displaying coccoid morphology with Gram stain. Additionally, well-demarked intracytoplasmic inclusion, known as Michaelis-Gutmann bodies were detected and stained positively with the Periodic acid-Schiff-diastase and Grocott stains. These findings were consistent with a diagnosis of pulmonary malakoplakia, a condition frequently associated with $R$ equi infection. The diagnosis was eventually confirmed by the culture results of BAL, where gram-positive coccobacillus grew on sheep blood and chocolate agar plates. Mycobacterial, yeast, and fungal cultures had negative results in BAL fluid. Blood cultures yielded negative results. On the basis of these histologic findings, the patient was admitted for treatment of the infection. Given the occlusion of a bronchus and the proximity of the lesion to the blood vessels of the pulmonary hilum, the patient was evaluated by a thoracic surgeon who suggested aconservative management because of the absence of recognizable signs of respiratory distress and bleeding. On day 53 , the patient started antimicrobial therapy that included intravenous meropenem ( $1 \mathrm{~g}$ twice daily) and oral azithromycin $(250 \mathrm{mg}$ 
once daily). Moreover, daily dose of TAC dose was reduced to total daily dose of $1.5 \mathrm{mg}$ (target TTL, $6-7 \mathrm{mg} / \mathrm{dL}$ ) in order to favor the resolution of the infection. The antibiotic therapy was well tolerated and yielded a gradual improvement of cough. After 15 days of treatment, antibiotic therapy was switched to oral levofloxacin (750 $\mathrm{mg}$ once daily) and oral azithromycin (250 mg once daily). Several days later, the patient was discharged while receiving the aforementioned antibiotic regimen that was planned until the resolution of disease. Serial chest CT scans, performed at 3 and 5 months from presentation, showed a progressive reduction in size of the perihilar lesion and a complete resolution of the consolidated area present in the right lower lobe. After a total duration of slightly more than 5 months of antibiotic therapy, when the size of perihilar lesion still measured $4 \times 13 \mathrm{~mm}$, the patient self-discontinued therapy for poor adherence. Given the high risk of relapse, the patient was warned to communicate every sign and symptom suggesting lung involvement. Fourteen months after the premature discontinuation of the antibiotic therapy, the patient remained asymptomatic, without developing any respiratory diseases or evidence of recurrence on chest radiography. She preserved a stable graft function with a creatinine of $1.9 \mathrm{mg} / \mathrm{dL}$ (estimated glomerular filtration rate, $25 \mathrm{~mL} / \mathrm{min} \mathrm{[5])} \mathrm{and} \mathrm{continued} \mathrm{an} \mathrm{immunosuppres-}$ sive regimen based on TAC (target TTL, 6-7.5 mg/dL) and methylprednisolone (4 $\mathrm{mg}$ once daily).

\section{DISCUSSION}

This case illustrates the complex management of $R$ equi pneumonia in a kidney transplant recipient affected by AIP. The main difficulties rely primarily on nonspecific clinical presentation, lack of a standard antimicrobial treatment, and presence of a severe underlying inherited disorder that significantly limited the antibiotic choice. $R$ equi is a gram-positive, obligate aerobic, nonmotile, and nonsporeforming organism responsible for rare but severe infectious disease in human beings. The major determinant factors of its pathogenesis are the capability to be a facultative intracellular microorganism, compromise host cell functions [6], and induce cell death [7]. The predominant route of transmission is thought to be the inhalation of infected aerosol and dust particles from the manure of herbivore animals [4]. Person-to-person transmission is infrequent; it was documented in 1998 when Arlotti et al [8] described an anecdotic case of transmission of the infection from a patient with $R$ equi pneumonia to 2 HIV-infected patients sharing the same hospital room. However, there are cases, as in our patient, in which it is impossible to determine the source of exposure. Intriguingly, this event is reported to be more frequent among immunocompetent patients than immunosuppressed patients.

$R$ equi is the cause of severe and often devastating disease, and if left untreated it can evolve into fatal systemic infection. $R$ equi infection has been described commonly among subjects with an altered immune system response, such as HIV-positive patients and transplant recipients.

Identification of the bacterium from the site of infection is the first step for successful management of the disease. To date, there are several different techniques to achieve the diagnosis. The conventional culture-based method provides information on phenotypic characteristics of culture (ie, growth pattern and colony morphology), whereas biochemical tests allow microbial identification by means of Christie-Atkins-Munch-Petersen reaction [9] and API Coryne system (BioMerieux, Marcy-l'Étoile, France) [10]. However, identification of $R$ equi from microbiological cultures is time-consuming as the bacterium has a long period of incubation that may require up to 7 days for identification [11]. On the other hand, polymerase chain reaction-based assay may be the most accurate and fastest diagnostic test for $R$ equi identification [12]. The major drawback, which limits its use, is its scarce availability outside university hospital and research centers [13]. Histologic examination is a useful tool to support the diagnosis of $R$ equi when malakoplakia is identified on the biopsy specimen. Malakoplakia is the typical histopathologic finding seen prevalently in patients with $R$ equi infection, especially during pulmonary involvement. This lesion is a rare acquired granulomatous disease, characterized by a dense infiltrate of foamy histiocytes containing concentric scattered basophilic inclusion, named Michaelis-Gutmann bodies, which likely represents the remnants of an impaired clearance of bacteria ingested by inflammatory cells [14]. Although malakoplakia is strongly associated with $R$ equi infection in immunocompromised subjects (especially in HIV-infected patients), it is a nonspecific lesion that has been also reported to occur in cases of Mycobacterium tuberculosis, Pasteurella multocida, and Escherichia coli infection [1]. From a practical point of view, the identification of malakoplakia together with gram-positive coccobacilli should raise a high index of suspicion for $R$ equi. Hence, as in our case, the collaboration with a skilled pathologist may be a critical prerequisite to identify the lesion and establish a presumptive diagnosis of $R$ equi infection, especially in a center like ours that is not equipped with polymerase chain reaction assay for detecting $R$ equi.

The most common presentation is necrotizing pneumonia, which may be complicated by abscess, empyema, pleural effusion, and spontaneous pneumothorax. Bloodstream invasion and peripheral organ dissemination are rare events and often secondary to lung involvement. Clinical manifestations of $R$ equi pneumonia are often subacute and nonspecific. Symptoms of pulmonary involvement were cough $(70 \%)$, chest pain (30\%), sputum production (20\%), and dyspnea (20\%) [15]. Radiologic findings of subjects diagnosed as having pneumonia are heterogeneous; dense infiltrates with upper-lobe cavitary lesion [16,17] often resemble pulmonary tuberculosis [18]. Occlusion of a bronchus is a rare manifestation of $R$ equi pneumonia, and only a few cases have been reported in literature to date [19-21]. In our case, the findings of lobar consolidation, lymphadenopathy, and bronchial obstruction associated with no clear signs of incipient infection disease were highly suggestive of lung cancer.

Antimicrobial therapy is the mainstay of treatment for $R$ equi infection. Treatment is usually based on multiple 
antibiotics; however, methods for determining in vitro susceptibilities are not standardized, and the choice of antibiotic is still essentially based on single-center experience. The bacterium is typically susceptible to erythromycin, rifampicin, ciprofloxacin, vancomycin, aminoglycosides, imipenem, and meropenem; it is usually resistant to penicillin and cephalosporin but extremely susceptible to amoxicillin/clavulanate and ampicillin/sulbactam. The sensitivity to tetracycline and sulfamethoxazole/trimethoprim is variable [22] and it seems to change between the United States and Europe [23]. The best treatment option is considered the inclusion at least of 1 antibiotic with intracellular penetration, such as erythromycin and/or rifampicin, in order to easily reach the microorganism within the host cells.

Treatment duration depends on the site, degree of tissue involvement, and underlying immunocompetence of the host; for instance, immunosuppressed patients with localization in the central nervous system and cavitary disease of the lung usually need a prolonged course of antibiotics. Transplant recipients need a long course of antibiotic therapy that include 2 to 6 week induction with an active intravenous antibiotic such as vancomycin, carbapenem, or aminoglycoside in combination with active oral drugs until a clear clinical improvement is established. After that, maintenance therapy based on 2 oral drugs continues until all culture results are negative and the symptoms and radiologic abnormalities have resolved. Commonly, pulmonary infection required at least 6 months of therapy [4]. Surgical intervention may be requested in cases of extensive or abscessual lesions [24,25]. The need for tapering or discontinuing immunosuppressive therapy in transplant recipients during $R$ equi is controversial $[24,26]$. This decision should be made in organ transplant recipients with a severe infectious disease and should be balanced against the everpresent risk of acute rejection.

Regarding the best treatment of $R$ equi infection in kidney transplant recipients affected by porphyria, data are lacking because there are only a few case reports of renal transplantation in patients affected by porphyria [27-30]. Porphyrias are a group of rare diseases, caused mainly by inherited enzyme activity defects of the biosynthetic heme pathway [31]. AIP is an autosomal dominant disorder caused by a defect in hydroxymethylbilane synthase, a heme biosynthetic enzyme. AIP attacks are characterized by severe abdominal pain, often accompanied by nausea, vomiting, tachycardia, and hypertension. The acute attacks may be complicated by neurologic manifestations (ie, neuropathy, confusion, coma) leading to potentially fatal consequences $[32,33]$. The identified precipitating factors are hormonal fluctuations, fasting, smoking, infections, and exposure to porphyrogenic drugs such as antibiotics. These conditions increase the concentration of 5-aminolevulinic acid and porphobilinogen in the liver that are thought to be neurotoxic [31]. Given this background, pharmacologic options for an infectious disease that require combination treatment may be limited in patients with porphyria. Being aware of the risks of some antibiotics to provoke an acute attack of porphyria, we selected them cautiously from a complete list of drugs available on the internet (for European Union countries) [34]. Among antimicrobials preferred for treating $R$ equi (Table 1 ), we avoided rifampicin, erythromycin, and sulfamethoxazole/trimethoprim because they are considered porphyrogenic; we avoided clindamycin and clarithromycin because they are likely to be porphyrogenic. Minocycline, cefazolin, kanamycin, and amoxicillin/sulbactam were also excluded because their risk of triggering an acute attack is unknown. Among drugs with a safer profile, we first used meropenem and then levofloxacin in association with azithromycin, an antibiotic with intracellular penetrance. The patient was treated with antibiotic therapy for a total duration of 5 months along with the withdrawal of MMF. This therapeutic strategy allowed the successful resolution of pneumonia without provoking acute attacks of porphyria or interactions with immunosuppressive drugs. Notwithstanding the reduction of immunosuppressive therapy, the patient did not develop an episode of graft rejection. Interestingly, antimicrobial therapy was discontinued prematurely when the disease was present radiologically, although in gradual resolution. After 15 months of follow-up, the patient had no signs of lung or systemic relapse. The delayed radiographic resolution of lung pneumonia, despite the clinical improvement, may open new questions about the usefulness of imaging in assessing resolution of pneumonia and guiding antimicrobial treatment duration. As with other forms of bacterial

Table 1. Antimicrobials Recommended for Treatment of Rhodococcus equi Infection in Patient Affected by Acute Form of Porphyria

\begin{tabular}{ll}
\hline Preferred Drug & Alternative Drug \\
Vancomycin (NP) & Amoxicillin-clavulanic acid (NP) \\
Tobramycin (NP) & Cefotaxime (PNP) \\
Gentamicin (NP) & Cefoxitin (PNP) \\
Ampicillin (NP) & Ceftriaxone (PNP) \\
Azithromycin (PNP) & Linezolid (PNP) \\
Ciprofloxacin (PNP) & \\
Levofloxacin (PNP) & \\
Imipenem and cilastatin (PNP) & \\
Meropenem (PNP) & \\
Drugs to Avoid & \\
Rifampicin (P) & \\
Erythromycin (P) & \\
Sulfamethoxazole/ & \\
$\quad$ trimethoprim (P) & \\
Clindamycin (PRP) & \\
Clarithromycin (PSP) & \\
Minocycline (NC) & \\
Cefazolin (NC) & \\
Kanamycin (NC) & \\
Amoxicillin/sulbactam (NC) & \\
\hline
\end{tabular}

The drugs are classified into 5 categories regarding their potential ability to precipitate symptoms: NC, not yet classified; NP, not porphyrinogenic; P, porphyrinogenic; PNP, probably not porphyrinogenic; PRP, probably porphyrinogenic; PSP, possibly porphyrinogenic. 
pneumonia, radiologic improvement during $R$ equi infection lags behind clinical improvement [35].

\section{CONCLUSION}

We describe the challenging management of a unique case of $R$ equi infection in a kidney transplant recipient affected by AIP. The careful selection of combination antibiotic therapy and reduction of immunosuppression allowed the successful treatment of pneumonia without triggering an acute attack of porphyria or an episode of acute rejection. We believe that use of levofloxacin, azithromycin, and meropenem can be safely administered for the treatment of $R$ equi infection in patients with AIP. Further studies are requested to provide guidelines for the optimal treatment regimen of $R$ equi infection.

\section{REFERENCES}

[1] Yamshchikov AV, Schuetz A, Lyon GM. Rhodococcus equi infection. Lancet Infect Dis 2010;10:350-9. https://doi.org/10.1016/ S1473-3099(10)70068-2.

[2] Takai S, Ohbushi S, Koike K, Tsubaki S, Oishi H, Kamada M. Prevalence of virulent Rhodococcus equi in isolates from soil and feces of horses from horse-breeding farms with and without endemic infections. J Clin Microbiol 1991;29:2887-9.

[3] Golub B, Falk G, Spink WW. Lung abscess due to Corynebacterium equi. Report of first human infection. Ann Intern Med 1967;66:1174-7.

[4] Weinstock DM, Brown AE. Rhodococcus equi: an emerging pathogen. Clin Infect Dis 2002;34:1379-85. https://doi.org/10.1086/ 340259.

[5] Levey AS, Stevens LA. Estimating GFR using the CKD Epidemiology Collaboration (CKD-EPI) Creatinine Equation: more accurate GFR estimates, lower CKD prevalence estimates, and better risk predictions. Am J Kidney Dis 2010;55:622-7. https:// doi.org/10.1053/j.ajkd.2010.02.337.

[6] Ferretti F, Boschini A, Iabichino C, et al. Disseminated Rhodococcus equi infection in HIV infection despite highly active antiretroviral therapy. BMC Infect Dis 2011;11:343. https://doi.org/ 10.1186/1471-2334-11-343.

[7] Zink MC, Yager JA, Prescott JF, Fernando MA. Electron microscopic investigation of intracellular events after ingestion of Rhodococcus equi by foal alveolar macrophages. Vet Microbiol 1987;14:295-305. https://doi.org/10.1016/0378-1135(87)90117-9.

[8] Arlotti M, Zoboli G, Moscatelli GL, et al. Rhodococcus equi infection in HIV-positive subjects: a retrospective analysis of 24 cases. Scand J Infect Dis 1996;28:463-7.

[9] Fernanandez-Garayzabal JF, Delgado C, Blanco MM, Suarez G, Dominguez L. Cholesterol oxidase from Rhodococcus equi is likely the major factor involved in the cooperative lytic process (CAMP reaction) with Listeria monocytogenes. Lett Appl Microbiol 1996;22:249-52.

[10] Giguère S, Prescott JF. Clinical manifestations, diagnosis, treatment, and prevention of Rhodococcus equi infections in foals. Vet Microbiol 1997;56:313-34. https://doi.org/10.1016/ S0378-1135(97)00099-0.

[11] Muscatello G. Rhodococcus equi pneumonia in the foalpart 2: diagnostics, treatment and disease management. Vet J 2012;192:27-33. https://doi.org/10.1016/j.tvj1.2011.08.009.

[12] Ladrón N, Fernández M, Agüero J, González Zörn B, Vázquez-Boland JA, Navas J. Rapid identification of Rhodococcus equi by a PCR assay targeting the choE gene. J Clin Microbiol 2003;41:3241-5.

[13] Rodriguez-Lazaro D, Lewis DA, Ocampo-Sosa AA, et al. Internally controlled real-time PCR method for quantitative species-specific detection and vapA genotyping of Rhodococcus equi. Appl Environ Microbiol 2006;72:4256-63. https://doi.org/10. 1128/AEM.02706-05.

[14] Stevens S, McClure J. The histochemical features of the Michaelis-Gutmann Body and a consideration of the pathophysiological mechanisms of its formation. J Pathol 1982;137:119-27. https://doi.org/10.1002/path.1711370205.

[15] Vergidis P, Ariza-Heredia EJ, Nellore A, et al. Rhodococcus infection in solid organ and hematopoietic stem cell transplant recipients. Emerg Infect Dis 2017;23:510-2. https://doi.org/10. 3201/eid2303.160633.

[16] MacGregor JH, Samuelson WM, Sane DC, Godwin JD. Opportunistic lung infection caused by Rhodococcus (Corynebacterium) equi. Radiology 1986;160:83-4. https://doi.org/10.1148/ radiology.160.1.3715049.

[17] Torres-Tortosa M, Arrizabalaga J, Villanueva JL, et al. Prognosis and clinical evaluation of infection caused by Rhodococcus equi in HIV-infected patients: a multicenter study of 67 cases. Chest 2003;123:1970-6. https://doi.org/10.1378/chest.123.6.1970.

[18] Gadkowski LB, Stout JE. Cavitary pulmonary disease. Clin Microbiol Rev 2008;21:305-33. https://doi.org/10.1128/CMR.0006007 . table of contents.

[19] Harvey RL, Sunstrum JC. Rhodococcus equi infection in patients with and without human immunodeficiency virus infection. Rev Infect Dis 1991;13:139-45.

[20] Sutor GC, Fibich C, Kirscher P, et al. Poststenotic cavitating pneumonia due to Rhodococcus equi in HIV infection. AIDS 1996;10:339-40.

[21] Hart DHL, Andrew JH, Peel MM, Burdon JGW. Lung infection caused by Rhodococcus. Aust N Z J Med 1988;18:790-1. https://doi.org/10.1111/j.1445-5994.1988.tb00182.x.

[22] Jacks SS, Giguère S, Nguyen A. In vitro susceptibilities of Rhodococcus equi and other common equine pathogens to azithromycin, clarithromycin, and 20 other antimicrobials. Antimicrob Agents Chemother 2003;47:1742-5. https://doi.org/10.1128/AAC. 47.5.1742-1745.2003.

[23] Gundelly P, Suzuki Y, Ribes JA, Thornton A. Differences in Rhodococcus equi infections based on immune status and antibiotic susceptibility of clinical isolates in a case series of 12 patients and cases in the literature. BioMed Res Int 2016;2016:2737295.

[24] Varotti G, Barabani C, Dodi F, et al. Unusual extrapulmonary Rhodococcus equi infection in a kidney transplant patient. Exp Clin Transpl 2016;14:676-8. https://doi.org/10.6002/ect. 2014.0176.

[25] Weinstock DM, Brown AE. Rhodococcus equi: an emerging pathogen. Emerg Infect 2002;34:1379-85. https://doi.org/10.1086/ 340259.

[26] Menon V, Gottlieb T, Gallagher M, Cheong EL. Persistent Rhodococcus equi infection in a renal transplant patient: case report and review of the literature. Transpl Infect Dis 2012;14:3-10. https://doi.org/10.1111/tid.12008.

[27] Nunez DJ, Williams PF, Herrick AL, Evans DB, McColl KE. Renal transplantation for chronic renal failure in acute porphyria. Nephrol Dial Transplant 1987;2:271-4.

[28] Barone GW, Gurley BJ, Anderson KE, Ketel BL, AbulEzz SR. The tolerability of newer immunosuppressive medications in a patient with acute intermittent porphyria. J Clin Pharmacol 2001;41:113-5.

[29] Warholm C, Wilczek H. Renal transplantation in a case of acute intermittent porphyria. J Clin Pharmacol 2003;43:1158-60. https://doi.org/10.1177/0091270003257223.

[30] Wahlin S, Harper P, Sardh E, Andersson C, Andersson DE, Ericzon B-G. Combined liver and kidney transplantation in acute intermittent porphyria. Transpl Int 2010;23:e18-21. https://doi.org/ 10.1111/j.1432-2277.2009.01035.x.

[31] Puy H, Gouya L, Deybach JC. Porphyrias. Lancet 2010;375: 924-37. https://doi.org/10.1016/S0140-6736(09)61925-5.

[32] Ventura P, Cappellini MD, Rocchi E. The acute porphyrias: a diagnostic and therapeutic challenge in internal and emergency 
medicine. Intern Emerg Med 2009;4:297-308. https://doi.org/10. 1007/s11739-009-0261-4.

[33] University of Modena and Reggio Emilia, Ventura P. The acute porphyric attack: a difficult diagnosis for a potential lethal event in emergency medicine. Emerg Med Trauma Surg Care 2015;2:1-10. https://doi.org/10.24966/ETS-8798/100005.
[34] The Drug Database for Acute Porphyria. http://www.drugsporphyria.org. [Accessed 17 July 2016].

[35] Mittl RL, Schwab RJ, Duchin JS, Goin JE, Albeida SM, Miller WT. Radiographic resolution of community-acquired pneumonia. Am J Respir Crit Care Med 1994;149:630-5. https://doi.org/ 10.1164/ajrccm.149.3.8118630. 